Annales Geophysicae (2002) 20: 757-769 (c) European Geophysical Society 2002

\title{
On the incidence of Kelvin-Helmholtz instability for mass exchange process at the Earth's magnetopause
}

\author{
R. Smets ${ }^{1,2}$, D. Delcourt ${ }^{3}$, G. Chanteur ${ }^{2}$, and T. E. Moore ${ }^{1}$ \\ ${ }^{1}$ NASA/Goddard Space Flight Center, Greenbelt, MD, USA \\ ${ }^{2}$ CETP/CNRS, Vélizy, France \\ ${ }^{3}$ CETP/CNRS, Saint Maur des Fossés, France
}

Received: 26 February 2001 - Revised: 21 January 2002 - Accepted: 6 February 2002

\begin{abstract}
Due to the velocity shear imposed by the solar wind flowing around the magnetosphere, the magnetopause flanks are preferred regions for the development of a KelvinHelmholtz instability. Since its efficiency for momentum transfer across the magnetopause has already been established, we investigate its efficiency for mass transfer. Using nonresistive magnetohydrodynamic simulations to describe the magnetic field shape in the instability region, we use testparticle calculations to analyse particle dynamics. We show that the magnetopause thickness and the instability wavelength are too large to lead to nonadiabatic motion of thermal electrons from the magnetosphere.

On the other hand, the large mass of $\mathrm{H}^{+}, \mathrm{He}^{+}$and $\mathrm{O}^{+}$ions leads to such nonadiabatic motion and we thus propose the Kelvin-Helmholtz instability as a mechanism for either magnetospheric ion leakage into the magnetosheath or solar wind ion entry in the magnetosphere. Test-particle calculations are performed in a dimensionless way to discuss the case of each type of ion. The crossing rate is of the order of $10 \%$. This rate is anti-correlated with shear velocity and instability wavelength. It increases with the magnetic shear. The crossing regions at the magnetopause are narrow and localized in the vicinity of the instability wave front. As a KelvinHelmholtz instability allows for mass transfer through the magnetopause without any resistivity, we propose it as an alternate process to reconnection for mass transfer through magnetic boundaries.
\end{abstract}

Key words. Magnetospheric physics (magnetopause, cusp and boundary layers; MHD waves and instabilities) - Space plasma physics (numerical simulation studies)

\section{Introduction}

The origin of magnetospheric plasma has been extensively studied using both in situ observations, theoretical models and numerical simulations. The ionosphere and the so-

Correspondence to: R. Smets (rsm@ @etp.ipsl.fr) lar wind represent the two possible sources, and their relative importance is still discussed. Several observations have demonstrated that the ionosphere supplies plasma to the Earth's magnetosphere (MSP) (e.g. Shelley et al., 1982; Chappell et al., 1987). Winglee (1998) revisited the concept of geopause proposed by Moore and Delcourt (1995) using 3-D multi-fluid simulations for different Interplanetary Magnetic Field (IMF) directions and showed that under southward IMF, the convection of ionospheric plasma out of the polar cap region is the major contribution to the plasma sheet population. The mass entry process from the solar wind can also supply magnetospheric plasma and many observations have been collected at the magnetopause (MP). The occurence of high-speed plasma flow has been interpreted as a signature of reconnection processes (e.g. Pashmann et al., 1979, 1986; Sonnerup et al., 1981; Gossling et al., 1982, 1991; Phan et al., 1996). Satellite crossings of the MP provided insights on its magnetic topology and on the particle distributions.

Using ISEE 1 and 2 measurements, Berchem and Russell (1982a) showed that the average thickness of the low-latitude dayside MP is about $800 \mathrm{~km}$. Le and Russell (1994) obtained a lower value for high $\beta$ (ratio of plasma pressure to magnetic pressure) in the magnetosheath (MSH). The average MP speed in the normal direction is a few tens of kilometers per second (e.g. Berchem and Russell, 1982a; Pashmann et al., 1993 ), and $\beta$ is about 1 , this value being larger (smaller) in the MSH (MSP) (e.g. Phan and Pashmann, 1996).

At the inner edge of the MP, the Low Latitude Boundary Layer (LLBL) was first defined by Eastmann et al. (1976) as the transition region between MSP and MSH (see also Mitchell et al., 1987). Using superposed epoch analysis of the AMPTE/IRM data, Phan and Pashmann (1996) found a thickness of the LLBL of $630 \mathrm{~km}$ that is uncorrelated with the $\beta$ value in the MSH. Mitchell et al. (1987) found a correlation between the thickness of the LLBL and the IMF direction. The magnetic topology of the LLBL is still unclear. Song et al. (1993) found that under northward IMF, the inner (earthward) part of the LLBL is on closed magnetic field 
lines, whereas the outer part could be composed of open and closed field lines. Fuselier et al. (1995) suggested that the LLBL could be on open field lines. A boundary layer is also observed at low latitudes in the MP flank (e.g. Eastmann and Hones, 1979). Mitchell et al. (1987) found that under northward IMF, this boundary is on closed field lines, but may lie on open or closed field lines for southward IMF.

The magnetic topology of the MP is of primary importance as it controls the mass loading process from the solar wind in the inner MSP. The first model of open magnetosphere proposed by Dungey (1961) invokes, at least locally, a component of the magnetic field normal to the MP. Examining EXPLORER 12 magnetic field measurements, Sonnerup and Cahill (1967) showed that in most MP crossings, the magnetic field component normal to the MP is very weak, and the magnetopause is thus essentially a tangential discontinuity (i.e. the magnetic field is always tangential to the MP). Studying the MP polarization, Berchem and Russel (1982b) also identified it as a tangential discontinuity, and showed a rotation of the magnetic field from its asymptotic value on both sides of the MP. Papamastorakis et al. (1984) obtained the same result for large field rotation angle and concluded that even for strong southward IMF (when reconnection is suspected to occur), the discontinuity is tangential and not rotational. Papamastorakis et al. (1984) concluded that the IMF direction is not the only parameter governing the reconnection process.

When MSP is closed, magnetic flux pile-up against MP first predicted by Midgley and Davis (1963) - and the resulting plasma depletion layer are observed (e.g. Pashmann et al., 1993; Song et al., 1993). Absence of magnetic flux pile-up is thus also interpreted as a consequence of the open structure of the MP (e.g. Phan et al., 1994). A statistical study of these accelerated plasma flows by Scurry (1994) showed that they occur preferentially for southward IMF, for large magnetic shear, at low $\beta$ value and in the subsolar region (see also Phan et al., 1996). Associated to these events, Phan and Pashmann (1996) found an enhancement of the parallel ion and electron temperatures when crossing the MP toward the LLBL.

Since the MP (and especially its flanks) is a preferred region for the development of Kelvin-Helmholtz (KH) instability, we investigate in this paper if nonadiabatic motion of charged particles in a $\mathrm{KH}$ instability can be at the origin of mass transfer through the MP. Therefore, we used $2 \mathrm{D}^{\frac{1}{2}}$ ideal MHD simulations to describe the magnetic field in the $\mathrm{KH}$ instability region, and test-particle computations to reconstruct the particle orbits. Considering the symmetry of the magnetic topology, the obtained results will be accounted and discussed as a mechanism for the escaping of ionospheric population from MSP to MSH, as the entry of solar wind in the MSP. Test-particle calculations are performed in a dimensionless way, and the results will be applied to both $\mathrm{H}^{+}, \mathrm{He}^{+}$ and $\mathrm{O}^{+}$ions. The basics of $\mathrm{KH}$ instability and its condition for occurence will be presented in Sect. 2. The methodology used to perform test-particle calculations will be described in Sect. 3. The obtained results will be generalized in Sect. 4 to a set of particles in order to derive macroscopic properties. Magnetospheric implications of these results will be discussed in Sect. 5.

\section{The Kelvin-Helmholtz instability}

The Kelvin-Helmholtz instability appears in a fluid flow in the presence of a shear velocity. In the classical hydrodynamic framework, there is no instability threshold for nonviscous fluid and a velocity shear is always unstable (e.g. Chandrasekhar, 1961). In magnetized plasmas, magnetic tension of the magnetic field lines tends to limit their bending and thus has a stabilizing effect. This yields an instability threshold (minimum value of the velocity shear) that depends on the initial conditions of the flow. It can easily be shown that, in the incompressible case, the wave vector of the instability in the direction normal to the interface is purely imaginary. The obtained perturbation is thus a surface wave localized at the interface.

Using the classical ideal MHD equations linearized at first order in the Fourier space $(\omega, \boldsymbol{k})$, the stability condition for the interface (equating the total pressure on each side of the interface) is

$\frac{\rho_{1}}{k_{N 1}}\left[\omega_{1}^{2}-\left(\boldsymbol{k} \cdot \boldsymbol{v}_{A 1}\right)^{2}\right]=\frac{\rho_{2}}{k_{N 2}}\left[\omega_{2}^{2}-\left(\boldsymbol{k} \cdot \boldsymbol{v}_{A 2}\right)^{2}\right]$,

where the subscripts 1 and 2 denote MSH and MSP, respectively; $\omega_{i}=\omega_{a}-\boldsymbol{k} \cdot \boldsymbol{v}_{i}$ is the Doppler shifted frequency in the media $i$ moving at velocity $v_{i}$, and $\omega_{a}$ is the frequency in the absolute frame. $\rho$ is the plasma density, $\boldsymbol{k}_{N}$ is the wave vector component normal to the interface and $\boldsymbol{v}_{A i}$ is the Alfvèn speed in media $i$. Equation (1) is obtained for an infinitely thin boundary. We thus have $k_{T 1}=k_{T 2}$ ( $k_{T}$ being the tangential component of the wave vector). The full MHD dispersion equation is

$$
\begin{aligned}
\omega^{4}- & \omega^{2}\left(k_{T}^{2}+k_{N}^{2}\right)\left(v_{S}^{2}+v_{A}^{2}\right) \\
& +v_{S}^{2} v_{A}^{2} \cos ^{2} \theta k_{T}^{2}\left(k_{T}^{2}+k_{N}^{2}\right)=0,
\end{aligned}
$$

where $v_{S}$ is the sound speed and $\theta$ is the angle between the tangential component of the wave vector and the magnetic field (e.g. Southwood, 1968). Assuming an incompressible media, $v_{S} \rightarrow \infty$ and Eq. (2) reduces to $k_{T}^{2}+k_{N}^{2}=0$, i.e. $k_{N}= \pm i\left|k_{T}\right|$. We thus have $k_{N 1}=-k_{N 2}$ and Eq. (1) reduces to (e.g. Fejer, 1964),

$$
\begin{aligned}
& \rho_{1}\left[\left(\omega-\boldsymbol{k}_{T} \cdot \boldsymbol{v}_{1}\right)^{2}-\left(\boldsymbol{k} \cdot \boldsymbol{v}_{A 1}\right)^{2}\right] \\
& \quad+\rho_{2}\left[\left(\omega-\boldsymbol{k}_{T} \cdot \boldsymbol{v}_{2}\right)^{2}-\left(\boldsymbol{k} \cdot \boldsymbol{v}_{A 2}\right)^{2}\right]=0 .
\end{aligned}
$$

The flow will thus turn unstable when solutions of the second degree equation in $\omega$ are imaginary. Thus, the instability criterion is

$$
\begin{aligned}
& \left(\boldsymbol{k}_{T} \cdot \Delta \boldsymbol{v}\right)^{2}>\left(\frac{1}{\rho_{1}}+\frac{1}{\rho_{2}}\right) \\
& \quad\left[\rho_{1}\left(\boldsymbol{k}_{T} \cdot \boldsymbol{v}_{A 1}\right)^{2}+\rho_{2}\left(\boldsymbol{k}_{T} \cdot \boldsymbol{v}_{A 2}\right)^{2}\right],
\end{aligned}
$$




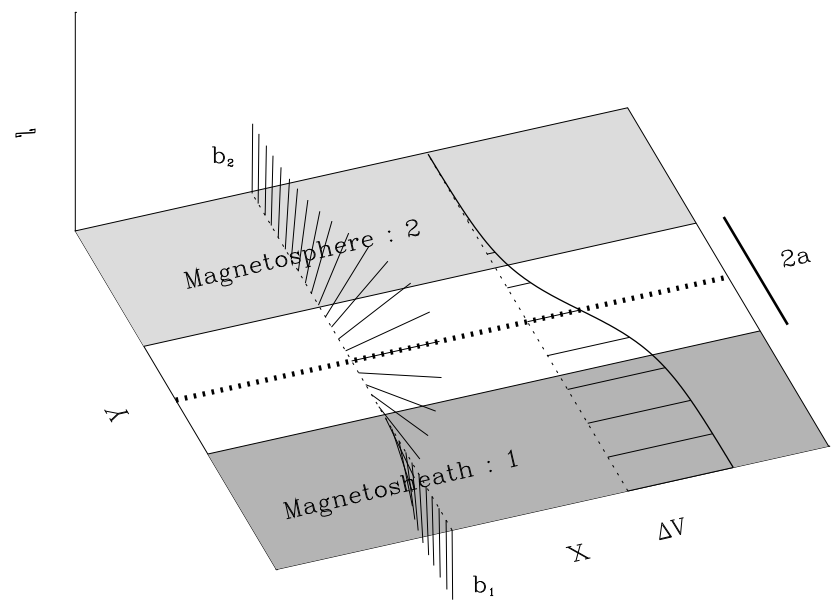

Fig. 1. The available TEC observations at $350 \mathrm{~km}$ altitude for a $15 \mathrm{~min}$ period.

where $\Delta \boldsymbol{v}=\boldsymbol{v}_{1}-\boldsymbol{v}_{2}$ is the shear velocity. Solutions of Eq. (4) have been discussed for different magnetospheric cases by Talwar (1964). Let us just emphasize two particular cases: (i) when the magnetic field is normal to the velocity shear, the flow is unstable regardless the value of the shear, (ii) when the magnetic field is parallel to the velocity shear and densities are equal on each side of the interface, the instability criterion is $\Delta v>2 v_{A}$.

The above results are obtained assuming an incompressible flow and an infinitely thin boundary. The incompressibility hypothesis implies that $v_{S} \gg v_{A}$, i.e. $\beta \gg 1$. However, one has $\beta \sim 1$ at the magnetopause (e.g. Pashmann et al., 1978). Taking into account the plasma compressibility, Fejer (1964) concluded that it reduces the stabilizing effect of the magnetic field. Using perturbative calculations, Sen (1964) put forward a stabilizing effect of the compressibility at large values of the velocity shear. Pu and Kivelson (1983) solved this paradox by computing Eq. (1) with Eq. (2). For a given value of $\theta$, they showed the existence of two solutions, namely quasi-slow and quasi-fast MHD modes (by analogy with the two MHD magnetosonic modes). At low values of the velocity shear an unstable mode thus appears, whereas the other mode is stabilized for high values of the shear (Eq. (1) impose a positive value of $k_{N}^{2}$ and the unstable surface wave evolves into a stable volume wave).

To take into account the thickness of the interface, the explicit form of the gradient in the normal direction has to be kept and the problem thus reduces to solving a second order differential equation (e.g. Walker, 1981). Miura and Pritchett (1982) studied the growth rate of the unstable mode depending on the wave vector $k$. In the incompressible case, Chandrasekhar (1961) showed that $\gamma \simeq k \Delta v$. Compressibility yields a bell-shaped curve, i.e. $\gamma$ increases with $k$ until a maximum value and then decreases to zero. Denoting $a$ as the half thickness of the interface, $\gamma$ is maximum for $2 k a \simeq 1$. An enhancement of the plasma compressibility leads to a decrease of both the growth rate $\gamma$ and the associated wave vector $k$.
Particle-like simulations have also been performed. Terasawa et al. (1992) used 2D hybrid simulations to issue the anomalous ion mixing in a KH instability. They showed that at the begining of the development of the $\mathrm{KH}$ instability, the mixing index (defined as the total area of the mixed cells normalized to the layer thickness) evolves as the square of time: $t^{2}$ phase. Then, the diffusion term due to the scattering of particles by the electric field (its non-electrostatic component) evolves as the square root of time. Thomas and Winske (1993) also used 2D hybrid simulations to put forward the inverse cascade (to longer wavelength) found in MHD and showed the existence of a small-scale structure (on the order of the ion larmor radius) that could be connected to flux transfer events or flux ropes. Two-dimensional Hybrid simulations used by Fujimoto and Terasawa (1994) showed that a large-scale mixing area develops at the corrugated interface. Three-dimensional hybrid simulations performed by Thomas (1995) showed results somewhat similar to 2D simulations and the strongly stabilizing role of the magnetic field is emphasized. It should be stressed here that in most particlein-cell simulations, emphasis is placed on particle mixing so that crossing rates cannot be thoroughly assessed. This is why we focused in this paper on the crossing particles, and try to find a good criterion to identify them.

We used a $2 \mathrm{D}^{\frac{1}{2}}$ MHD code (Belmont and Chanteur, 1989) to compute the magnetic field in the $\mathrm{KH}$ instability region. This code resolves the classical MHD equations with a polytropic law for the closure equation. Due to numerical resistivity, the magnetic Reynolds number $\mathrm{R}_{M}$ cannot be larger than 8000. The simulations used in this study were performed with $\mathrm{R}_{M}=2000$. Under southward IMF, the MP has the structure of a current sheet (e.g. Cahill and Amazeen, 1963, Berchem and Russell, 1982b). The initial geometry of the MP used for MHD simulations is sketched in Fig. 1. Subscripts 1 and 2 stand for MSH and MSP, respectively, and $a$ is the MP half thickness. The $X$ axis is along the Sun-Earth direction toward the tail (this direction being periodic); the $Z$ axis is in the north-south direction toward the north, which is also the invariant direction, and the $Y$ axis is in the dawndusk direction toward dawn. Figure 1 thus depicts the dusk side of the MP. We studied a rotation of $180^{\circ}$ of the magnetic field which corresponds to a southward IMF. Even if systematic calculations have been performed in this case, different polarization of the MP will be discussed in Sect. 5. Defining $\chi=0.5 \times\left[1+\tanh \left(\left(y-y_{0}\right) / a\right)\right]$, where $y_{0}$ is the position where the $Z$ component of the magnetic field vanishes, we have $\alpha=\alpha_{1}+\chi\left(\alpha_{2}-\alpha_{1}\right)$ as the local direction of the magnetic field relative to the $X$ axis. We define locally the initial density, velocity and magnetic field as

$$
\begin{aligned}
& \rho=\rho_{1}+\chi\left(\rho_{2}-\rho_{1}\right), \\
& v=v_{1}+\chi\left(v_{2}-v_{1}\right), \\
& b_{T}=b_{1}+\chi\left(b_{2}-b_{1}\right) .
\end{aligned}
$$

The three components of the magnetic field are given by $b_{x}=b_{T} \cos \alpha, b_{y}=0$, and $b_{z}=b_{T} \sin \alpha$. We used a $512 \times 512$ grid in the $X-Y$ plan. The simulation box size 
is $2 \pi$ in the $X$ direction and $\pi$ in the $Y$ direction. Results are presented in normalized coordinates, namely lengths are normalized to ten times the MP thickness, velocities are normalized to the MSH Alfvèn speed and the magnetic field is normalized to the asymptotic magnetic field magnitude in the MSH.

With such an initialization of the MP structure, we do not take into account the inner part of the LLBL. Phan and Pashmann (1996) showed that for high magnetic shear, most of the magnetic field rotation occurs at the outer edge of the LLBL. The inner edge of the LLBL (bordering the MSP) is a region where both electron and ion temperatures can increase sharply, but the magnetic field weakly changes. In the following, we will focus on the magnetic field structure. This allows us to simplify the problem by decreasing the number of initial parameters.

Figure 2 presents the evolution of the magnetic pressure through time in the $X-Y$ plane. Dotted lines in each panel represent the boundary between positive and negative $Z$ components of the magnetic field. We will use this boundary as the definition of the MP position. The initial conditions in Fig. 2 are $\Delta v=2 v_{A 1}, \beta_{1}=2, b_{1}=b_{2}, \alpha_{1}=90^{\circ}$, $\alpha_{2}=270^{\circ}, \rho_{1}=1$, and $\rho_{2}=0.1$ The four panels present, from top to bottom, the magnetic pressure at $t=10, t=12$, $t=14$ and $t=19$, respectively. At the beginning of the simulations $(t=10)$, the interface is fairly stable, but the magnetic pressure strongly decreases in its vicinity (while it was initially uniform). At $t=12$, the instability is fully developed and the wave front is quite steep. Even if the plasma is in a nonlinear state, the magnetic field is fairly structured. At $t=14$, the wave front starts breaking and eddies merge together. At the end of the simulation $(t=19)$, one can see the result of an inverse cascade for which small eddies gather and give their energy to a larger one. The resulting dominant wavelength is larger than the one observed when the instability was rising at $t=12$. The vortex pairing in the nonlinear evolution of the $\mathrm{KH}$ instability has already been pointed out in MHD simulation (see, e.g. Belmont and Chanteur, 1989), and studied recently by Miura (1999). Of course, this inverse cascade is limited by the size of the simulation box. Since boundary conditions are periodic, the KH instability wavelength cannot be longer than the $X$ size of the simulation box. This is the limitation of all localized studies and cannot be resolved unless the instability is studied with a large-scale or global simulation. But this is not a limitation for this study, since the shear velocity barely modify the results. This point will be raised in Sect. 5 .

These MHD results show that the development of the KH instability leads to a bending of the magnetic field lines in the vicinity of the interface. To explore the nonadiabatic regime of charged particles in the $\mathrm{KH}$ instability, one may compute the $\kappa$ parameter defined by Büchner and Zelenyi (1989). However, detailed analysis of the particle orbits (see Sect. 3) reveals that nonadiabatic motion essentially arises from the small value of the MP thickness, rather than from the curvature of the magnetic field lines induced by the $\mathrm{KH}$ instability. This is emphasized by the vanishing of the mag-
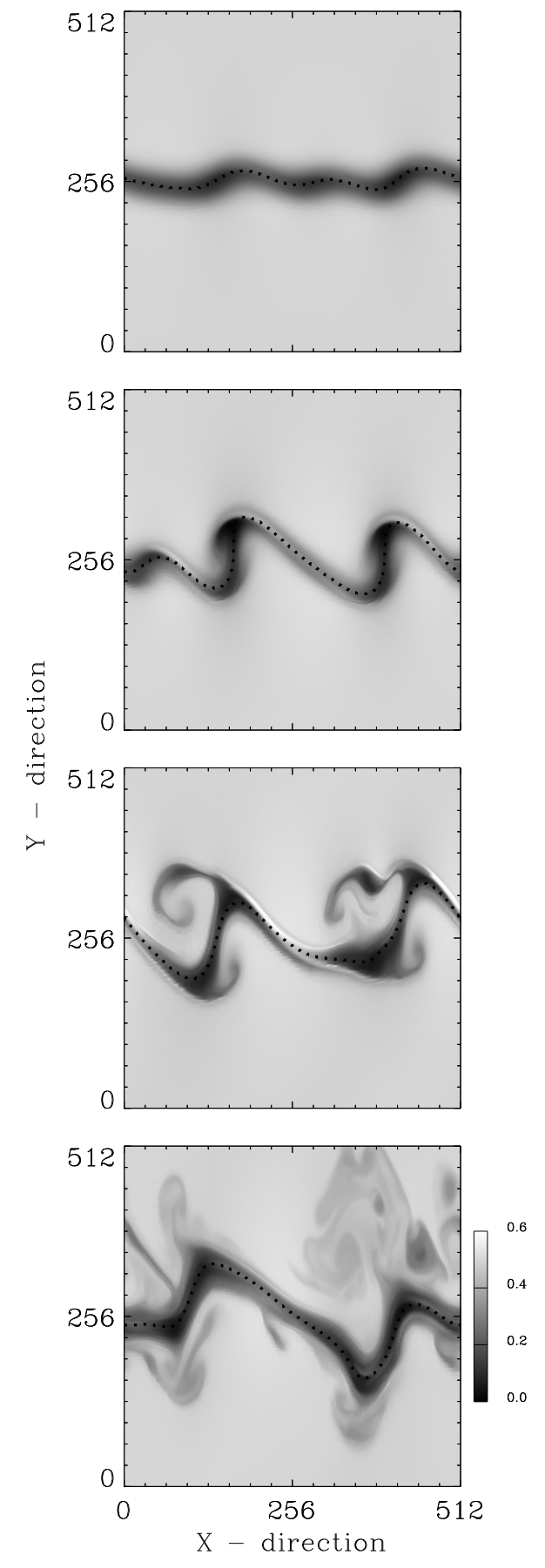

Fig. 2. Color-coded evolution of the magnetic pressure in the $X-Y$ plane from top to bottom: at $t=10, t=12, t=14$, and $t=19$.

netic field magnitude close to the MP, with the particle Larmor radius being inversely proportional to it.

Since the KH instability is a good mechanism to ensure momentum transfer from MSH to MSP (e.g. Miura, 1987), its efficiency for mass entry from the solar wind or escaping from MSP is investigated. Systematic calculations for electrons demonstrated that these particles behave most of the time adiabatically and that the $\mathrm{KH}$ instability is unable to induce nonadiabatic MP crossing of these particles. The 
main reason is that, regardless of the $\mathrm{KH}$ instability state of development, the MP is too thick to break the adiabaticity of the electron motion and induce anomalous diffusion. Even if the magnetic pressure drastically decreases at the MP, the associated Larmor radii of electrons located in this region are small compared to the thickness of the magnetic field reversal layer. Furthermore, the evolution time scale for the instability is large compared to their gyroperiod. Thus, electrons verify the MHD hypothesis of low frequency and long wavelength. Due to the larger mass of $\mathrm{H}^{+}, \mathrm{He}^{+}$and $\mathrm{O}^{+}$ions, they experience nonadiabatic motion in the vicinity of the $\mathrm{KH}$ instability. Thus, one may wonder whether the $\mathrm{KH}$ instability allows for a substantial crossing of these ions through the MP. Hereinafter, calculations are performed in a dimensionless way, allowing for a discussion of these different types of ions in Sect. 5.

\section{Single-particle dynamics}

Tracing particle orbits in MHD fields calls for a few cautions. Since the magnetic field is defined on each grid point, interpolation is needed to compute the field in between. In the following, we chose a bilinear interpolation in order to ensure $\boldsymbol{\nabla} \cdot \boldsymbol{b}=0$. The magnetic field is thus continuously defined, but not its gradient. Some numerical gradient drift can thus appear when the particles reach the edges of a cell. However, calculations reveal that this happens so seldom that it does not significantly affect the results. Quadratic interpolation may avoid such numerical artifacts, but the magnetic field divergence would then be nonzero.

Particles dynamics is also constrained by the fluid flow yielding to the electric field $\boldsymbol{e}=-\boldsymbol{v} \times \boldsymbol{b}$. Since the instability is nonstationary, as the position of the MP, a temporal interpolation is needed to describe the time evolution of the electromagnetic fields. This type of calculation has been performed and showed that the temporaly interpolated electric field gave rise to large numerical instabilities as the flow velocity and the magnetic field sharply reverse at the MP. Even small inaccuracies in the electric field values yield to large incorrect energy gains for particles. Focusing on the time scale needed for particles to cross the MP, it is small compared to the time scale of the instability evolution, since the $\mathrm{KH}$ instability phase velocity is small compared to the particle velocity. This point will be discussed in further detail in Sect. 5, but at this point we consider that the calculations can be performed by neglecting the time evolution of the instability without significantly impacting the results.

Systematic orbit calculations in the magnetic field topology obtained in the third panel from top of Fig. $2(t=14)$ were performed in a dimensionless way. The particle motion equation becomes $d_{t} \boldsymbol{v}=\boldsymbol{v} \times \boldsymbol{b}$ when the magnetic field is normalized to the asymptotic magnetic field in the MSH, where the time is the inverse of the gyroperiod and the length is ten times the MP thickness. This normalization is different from the one used for the MHD simulation and the ratio between the two, with normalized time and velocity, will be discussed in Sect. 5 on the basis of realistic values of magnetic field magnitude and density in the MSH.

Particle motion was computed over 50 gyroperiods (calculated using the asymptotic value of the magnetic field magnitude in the MSH). Particles were initially in the MSH and we focused on the one crossing the MP in order to end up in the MSP. Due to the symetry of the magnetic field topology, it was verified that the same type of dynamics was obtained for particles initially in the MSH and in the same proportion. This is also a consequence of conducting the calculations in the wave frame. The choice of 50 gyroperiods is large enough to ensure mass transfer through the MP and small enough for the steady state assumption to be valid. Increasing the maximum time for the simulation to 100 gyroperiods increases the rate of crossing particles, but not in a significant way. Transient crossings of the MP that last a few gyroperiods can be observed when following particle orbits for a long enough time. We then have to define when a particle can be considered as having crossed the MP and when it is just meandering in its vicinity. The criterion for a particle to be considered as a crossing one is to initially perform 3 cyclotron turns on one side of the MP, and 3 cyclotron turns as well once it has crossed the MP. This allows one to discard transient crossings of the MP associated with meandering motion in its vicinity. In the same way, increasing this minimum value of cyclotron turn, initially in the MSP and finally in the MSH, decreases the Crossing Rate (CR). These kinds of numerical constraints always play a role in computations and prevent any sort of sharp quantitative analysis. But the sensibility to these parameters is gentle enough to allow for a qualitative interpretation of the results, as presented below.

To investigate the dependence of the $\mathrm{CR}$ on the state of development of the KH instability, systematic computations have been performed using the MHD electromagnetic field at different times. These calculations were performed without any temporal interpolation, assuming that the MHD electromagnetic fields were stationary. These calculations have been performed with a given initial energy, namely 0.05 , a given initial pitch-angle, namely $60^{\circ}$ and a sampling of the whole $X-Y$ plan using a $80 \times 80$ grid. At each grid point (thus different from the grid used for the MHD simulations), the gyrophase domain is sampled with 6 different initial gyrophases uniformly distributed. Further test calculations reveal that a larger number of initial gyrophases does not change the results.

The computations were performed every half time step of the MHD simulations and the results are displayed in Fig. 3. The CR value is defined as the ratio between the number of crossing particles to the number of particles located at less than two Larmor radii from the MP position. As a matter of fact, particles that are initially too far from the MP will never reach it, since the magnetic field is uniform and unbended and thus, the particles have no chance of crossing the MP. To be significant, the crossing rate needs to be normalized to the number of particles that are potentially able (because they are close enough) to cross the MP. Until $t=24$, 


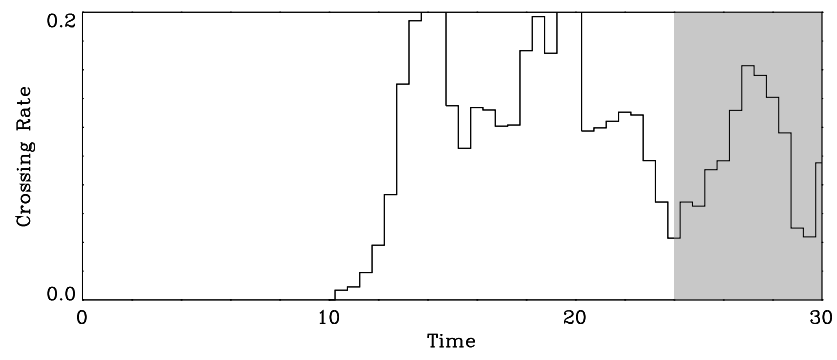

Fig. 3. Number of escaping particles depending on normalized time.

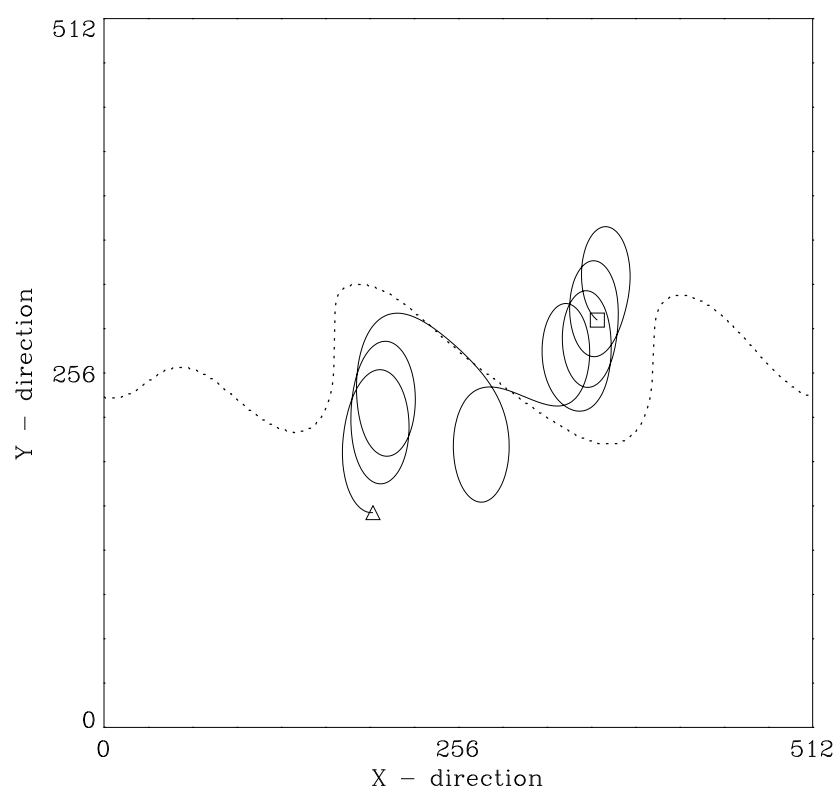

Fig. 4. Particle orbit crossing the interface in a nonadiabatic manner. The test particle starts in the MSP (square) and ends up in the MSH (triangle).

the average value of the $\mathrm{CR}$ is $12 \%$ and its standard deviation is $5 \%$. We do not take into account the results obtained for $t>24$ (shaded region in Fig. 3), because mass loss occurs at the $Y$ boundaries and the MHD electromagnetic field becomes inaccurate. An important aspect is that the CR remains fairly constant, whereas the instability wavelength increases with time. Calculations with higher shear velocity (implying higher wave length) confirm this anti-correlation between the CR and the instability wave length. This point will be discussed in Sect. 5. Also, one has to notice that the use of a $2 \mathrm{D}^{\frac{1}{2}}$ code is justified, since particles remain close enough to the equatorial plane (at less than $3 R_{E}$ using standard values of the MP thickness for the normalization). A larger extension of the motion in the $Z$ direction would call for a better description of the magnetic field lines bending at high latitude.

To illustrate the type of computed orbits, Fig. 4 depicts the one obtained for a positively charged particle launched with an energy of 0.05 and a pitch-angle of $60^{\circ}$ from the MSP (indicated by a square) and ending up in the MSH (indicated by

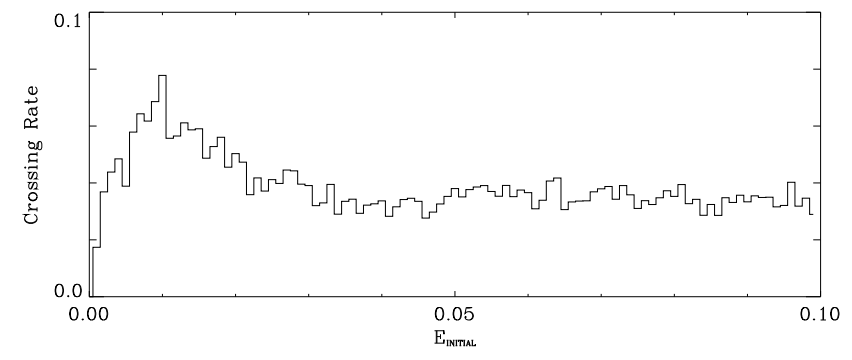

Fig. 5. Number of escaping particles depending on initial energy.

a triangle). The obtained orbit is representative of a general behavior. The particle has initially an adiabatic motion in the MSP. The steep gradient of the magnetic field magnitude at the MP brings forth strong deformation of the cyclotron turns. Then, the particle comes close enough to the MP and crosses it. Due to the magnetic field reversal at the MP, the gyromotion on the other side of the MP occurs in the opposite direction. Furthermore, since the magnetic field gradient is still steep, the gyromotion is highly distorded. But the three full cyclotron turns can be clearly seen in the MSH, thus satisfying the test to stop the computation. This orbit is representative of the one for which the crossing of the MP is brief, since the process occurs in less than 10 gyroperiods. Other orbits will be presented that exhibit transient motion at the MP before definitively crossing it.

Under the above assumptions, we can now examine the dependence of the CR with initial energy and pitch-angle. Since the convection electric field is not considered, the particle energy remains constant. The use of a 4th order RungeKutta scheme (for the particle motion integration) provides enough accuracy for the artificial energy variation due to the fact that the numerical technics are smaller than $0.01 \%$. Figure 5 presents the variation of the CR with initial energy. The CR increases with energy to reach its maximum value at $E=0.01$. Then, it decreases until $E=0.02$, to reach $\mathrm{CR}=$ 0.04. After this threshold, the CR is somewhat constant regardless of the initial energy of the particles. The importance of the normalization technics needs to be discussed. As mentioned above, the $\mathrm{CR}$ is calculated using a normalization to the number of particles initially located at less than 2 Larmor radii from the MP. Since the Larmor radii depend on the particle energy, the size of this nonadiabatic sheet will decrease with energy. The population or depletion of this nonadiabatic sheet would need to resolve the plasma motion upstream of the MP.

As mentioned above, the calculations presented in this paper have been performed by neglecting the fluid velocity compared to the particle velocity. Since the associated $\boldsymbol{v} \times \boldsymbol{b}$ electric field is also neglected, the particle energy remains constant. Hence, all crossing particles will carry their momentum themselves. The CR equal to 0.04 , obtained in Fig. 5, is thus also an estimation of the momentum transfer. It is interesting to notice that it is somewhat the same as the one obtained by Miura (1987), even if the physical 


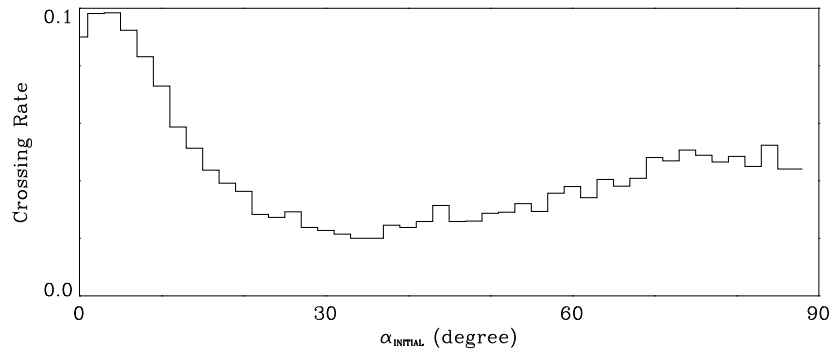

Fig. 6. Number of escaping particles depending on initial pitchangle.

process is somewhat different. In the work of Miura (1987), the anomalous (eddy plus magnetic) viscosity is responsible for this momentum transfer by enhancing the initially null particle velocity at the discontinuity. In the present study, the crossing of the particles is responsible for the transfer. It must also be noted that this rate is calculated with a very different method of normalization, since Miura (1987) calculated the peak of the spatial average over one wave period of the tangential velocity. This also raises the difficulty of having a crossing rate integrated over the whole cross section of the magnetosphere, unless one uses a global simulation.

Figure 6 presents the evolution of the CR with the initial pitch-angle value $\alpha$. These calculations are conducted using an initial energy of 0.05 . For initially field-aligned particles, the $\mathrm{CR}$ decreases as the initial pitch-angle increases, until it reach $30^{\circ}$. The $\mathrm{CR}$ is thus at its minimum value, namely 0.02 and then from $30^{\circ}$ to $90^{\circ}$ of initial pitch-angle value, the CR increases until about 0.04 . There exists a smooth dependence of the CR with the initial value of the pitch-angle. But this value does not drastically evolve, and furthermore, the profile obtained in Fig. 6 becomes flatter and less pronounced when using a larger value for the minimum number of cyclotron turns. For the calculations presented below, $\alpha=60^{\circ}$ will be used as the initial value. On the other hand, these particles will be pitch-angle scattered when crossing the MP. The consequences of this individual behaviour for a large collection of particles is discussed in the next section.

\section{Macroscopic implications}

Figure 5 showed that the $\mathrm{CR}$ is somewhat constant depending on the initial energy of the particles. But since this rate is normalized to the number of particles located at less than 2 gyroradii from the MP, the number of concerned particles decreases with their initial energy. Even if some process can account for bringing particles close to the MP, the distribution function resulting from the crossing particles is expected to have a weaker density at low energy compared to that at higher energy; hence, there is a build-up of a high energy tail distribution, which is beyond the scope of this paper and would need to take into account the energy gain of the particles via the electric field.

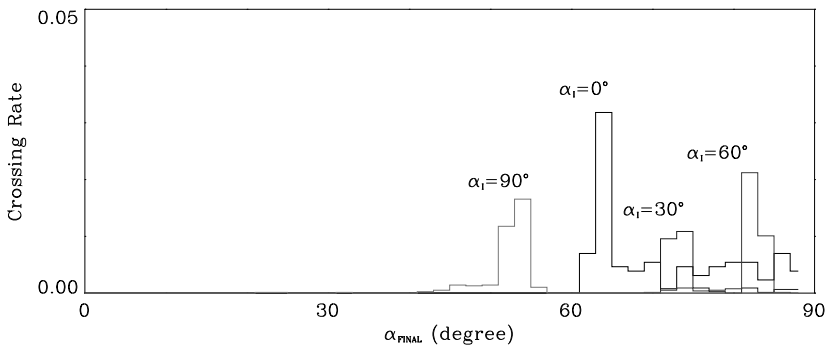

Fig. 7. Number of particles crossing the MP depending on final pitch-angle at the observation point. The four curves correspond to the initial pitch-angles that are labeled.

On the other hand, pitch-angle scattering upon crossing of the MP leads to a clear structuring of the phase space. This can be appreciated in Fig. 7, which presents the CR depending on the pitch-angle at the observation point after crossing the MP. These computations was performed by sampling the whole initial pitch-angle domain with a step of $2^{\circ}$. In order not to cloud the results, only 4 curves, corresponding to $0^{\circ}, 30^{\circ}, 60^{\circ}$ and $90^{\circ}$ initial pitch-angle (indicated by the text and different levels of grey), are displayed. As the magnetic field magnitude is constant across the MP, the projection of the particle pitch-angle on an asymptotic magnetic field is not a problem. Furthermore, the test described in Sect. 3 ensures that particles are in an adiabatic sequence when the orbit tracking is interrupted so that the pitch-angle can be accurately defined.

Focusing on one of these curves, it is clear that the obtained distribution is highly collimated along a specific direction, which depends on the initial pitch-angle. For zero initial pitch angle, the distribution obtained after interaction with the MP will be collimated at about $60^{\circ}$ from the magnetic field direction. As the initial pitch-angle increases, the angle formed by the obtained beam with the direction of the magnetic field will also increase, until it reaches $90^{\circ}$ for the initial distribution at approximately $75^{\circ}$. Then the obtained beam is located at $50^{\circ}$ and continues to drift until $60^{\circ}$ as the initial pitch-angle of the distribution reaches $90^{\circ}$. As displayed in Fig. 6, the CR depends on the initial pitch-angle. This is the reason why 4 peaks are obtained in Fig. 7.

There exists a clear bijective relation between the initial and final particle pitch-angles after crossing the MP. To illustrate the pitch-angle distribution obtained from an initially isotropic population, Fig. 8 shows the average of the curves presented in Fig. 7 (using the wholes curves obtained with a $2^{\circ}$ step). Since the distributions obtained in Fig. 7 are sharply collimated, a good sampling is needed to smooth the obtained peaks. Figure 8 clearly depicts the emergence of a population mainly in the direction perpendicular to the magnetic field. A better sampling would certainly smooth the contours, but will not change the overall direction of this distribution. Particle interaction with a $\mathrm{KH}$ instability is thus an efficient mechanism to pitch-angle scatter an initial population in a specific direction, producing for a $180^{\circ}$ magnetic shear a population in the direction perpendicular to the 


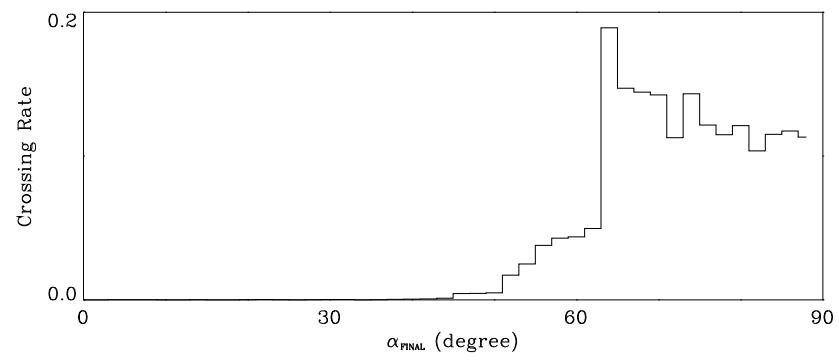

Fig. 8. Number of particles crossing the MP depending on final pitch-angle and assuming an initially isotropic distribution.

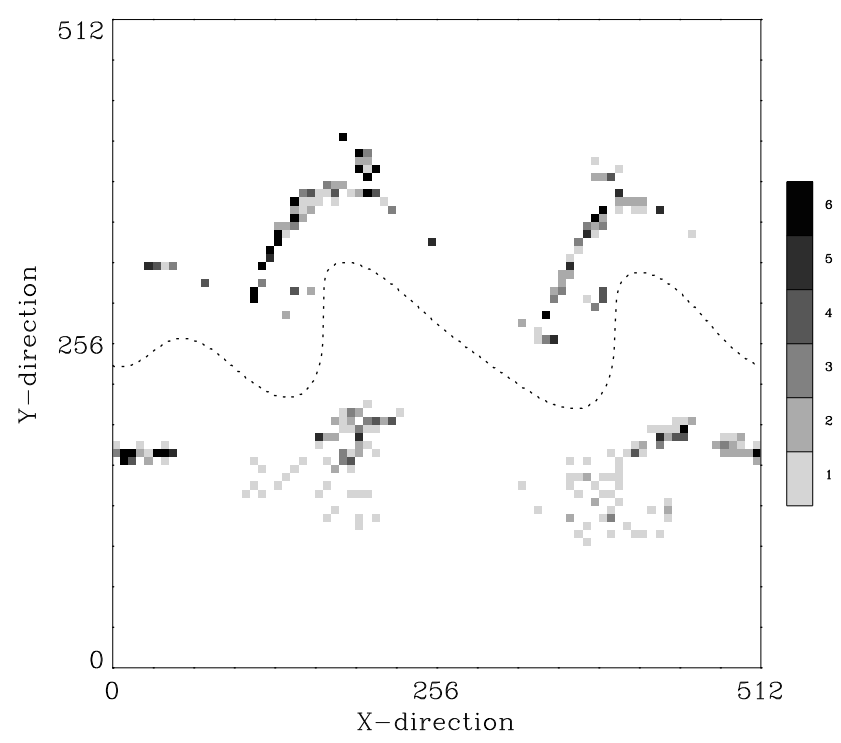

Fig. 9. Color-coded number of particles crossing the MP. Initial and final position in the $X-Y$ plane is depicted.

magnetic field.

This structuring in velocity space goes together with structuring in position space. This can be appreciated in Fig. 9 which presents the initial and final positions of the crossing particles. As mentioned above, for each grid point the orbit of 6 particles is computed by sampling the whole initial gyrophase space.

Figure 9 shows the number of particles crossing the MP. The color-code used depicts the number of particles (from 1 to 6) that cross the MP while their location, both initial and final, is represented by each pixel position. Remembering that particles initially start in the MSP side of the MP, the points displayed at large $Y$ values are the initial positions and the parts displayed at low $Y$ values represent their coresponding final positions. In this figure, one can clearly see islands of escaping particles localized near the edge of each wave front, while they end up narrowly localized near the steep part of the wave profile on the other side of the MP.

The feature displayed in Fig. 9 Triggers the question of the time evolution of the escaping regions. Terasawa et al. (1992) put forward the existence of a $t^{2}$ phase, describing the time evolution (at the early stage of the instability) of

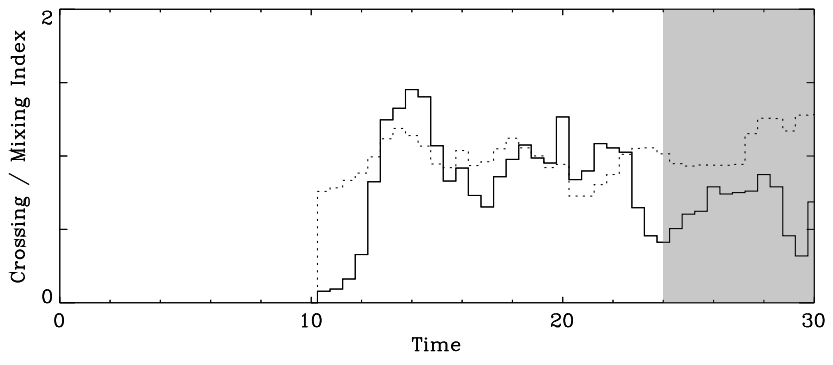

Fig. 10. (Solid line) crossing index and (dashed line) mixing index depending on normalized time.

the mixing layer. Their mixing index (representing the size of the mixing region with a suitable normalization) showed a quadratic evolution with time. In order to compare our results with this one, we computed in Fig. 10 the time evolution of the crossing index (solid line) and the mixing index (dashed line), using the same normalization. For the clarity of the figure, the mixing index is divided by 10 . The mixing index is the size of the mixing layer. In a like manner to Terasawa et al. (1992), we consider that a cell is mixed if the fraction of ions that cross it is at least equal to 0.25 . The crossing index is the same one with the constraint on each ion to stay at least 3 gyroperiods in the MSP after crossing. It is clear that neither of these curves depict a $t^{2}$ phase. Thomas and Winske (1993) found a linear phase that was also different from the $t^{2}$ phase, and argued that the different initial topologies used are based on the origin of this discrepency. This argument certainly holds also in the case of this paper, as we considered gradients in density, magnetic field magnitude and magnetic field direction.

The evolution of the curves displayed in Fig. 10 are also quite different from the regular and smooth evolution obtained in the two papers cited above. In test-particle simulations, all particles have a different time-of-flight, which is different from the case of self-consistent, particle-like simulations. To build a map of mixing regions would thus be meaningless in test-particle calculations and the dashed line in Fig. 10 can hardly be compared to the results of Terasawa et al. (1992). On the other hand, the crossing index depicted by the solid line provides some new insights on the mass transfer induced by $\mathrm{KH}$ instability. The difference in the particles' time-of-flight is not a problem, since all the crossing particles reach a stationary state in the sense that they stay on the MSP side of the MP. Such a conclusion could not be reached from a snapshot of the mixing region at a given time. Indeed, self-consistent particle-in-cell simulations generally display instantaneous fields contrained by the existence of a $t=0$ time. The curves displayed in Fig. 10 thus lead to a conclusion: there is about one order of magnitude between the crossing rate and the mixing rate, meaning that most of the particles trapped at the boundary never cross the MP in a definitive way.

The localization of the entry regions depicted in Fig. 9 is of primary importance for the observation of such a population. 
This means that in addition to the signature associated with the magnetic field structure, there also exists a signature in the particle distribution. The crossing particles, regardless of the direction of the crossing, are located in the close vicinity of the wave front associated with the KH surface wave. Even if the large-scale motion of plasma is suspected to smooth and merge these bubbles, the stationarity of the process maintains their structure. For a spacecraft traversing the MP under $\mathrm{KH}$ instability, the spatial localization of escaping points in the MSH suggests the occurence of particle bursts near the interface. For a spacecraft orbit tangent to the MP, observation of such bursts should provide the location of the steepened part of the profile. On the other hand, crossing of the MP in the transverse direction, far from the wave front, will not allow for observation of such bursts.

To conclude this section, there exists a clear structure of the plasma in the vicinity of a KH wave. The efficiency of the process does not depend on the initial energy of the particles and the number of particles concerned increase with their energy. The larger the particle energy is, the larger the number of particles crossing. Even if the structure of the distribution function cannot be issued, this process leads to the build-up of a high energy tail distribution. On the other hand, there is a clear structure in the pitch-angle distribution. The particles crossing the MP are mainly in the perpendicular direction after their crossing of the MP. This result is limited to the $180^{\circ}$ magnetic shear case. The crossing particles are initially located at the edge of the wave front and after crossing of the MP, end up at the edge of the KH wave on the other side.

\section{Discussion}

Examination of crossing particle orbits allows us to better understand the escape process. Figure 11 depicts the orbit of an ion initially in the MSP (position indicated by the square) and then entering into the MSH (indicated by the triangle). One can identify two adiabatic sequences at the beginning and at the end of the orbit, a meandering sequence near the interface and transition sequences in between. Meandering sequences are somewhat similar to those predicted by Speiser (1965). Due to the sharp field reversal across the interface on a length scale comparable to the particle Larmor radius, particles execute about half a cyclotron turn on each side of the MP. These turns occur in opposite directions due to the antiparallel geometry of the magnetic field. In a recent paper, Smets (2000) discussed the nonlinear dynamic of charged particles in such magnetic topology, and put forward the similarities and differences between a Harris sheet and a tangential discontinuity.

The orbit of Fig. 11 exhibits a sequence (around $X=128$ ) where the particle experiences a nearly full cyclotron turn on each side of the MP. The resulting figure eight-like orbit looks similar to the one discussed by Sonnerup (1971) (see, e.g. Fig. 2 of Sonnerup, 1971). This type of orbit follows from the sharp field reversal at the interface. Near $X=256$, Fig. 11 also exhibits a sequence where the particle experi-

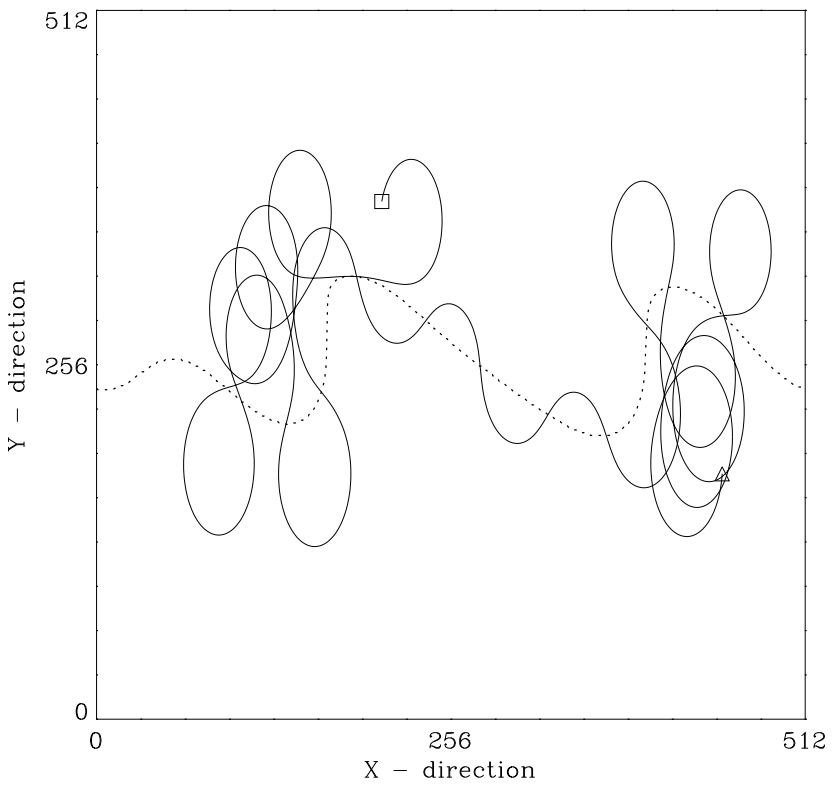

Fig. 11. Particle orbit crossing the interface. The particle starts in the MSP (square) and ends up in the MSH (triangle). Both Speiserlike and figure eight-like sequences can be observed.

ences just half a cyclotron turn on each side of the MP. The resulting Speiser-like orbit resembles the one discussed by Speiser (1965) in a Harris sheet. It has to be noted, however, that without any KH instability, these types of orbits are regular. As a matter of fact, it has been shown numerically by Smets (2000) that the action integral $I_{z}=\int \dot{z} d z$, where $z$ is the direction of fast oscillation, is conserved during such a motion. In a similar manner to dynamics in a Harris sheet, each particle orbit may have crossing (meandering at the MP) or non-crossing sequences, depending on the initial position in phase space.

For non-crossing (crossing) sequences, a magnetic moment (action integral) will be conserved and the particle motion is regular. On the other hand, it has been shown that in a modified Harris sheet, chaos arises when a small component of the magnetic field normal to the current sheet is added (see, e.g. Chen and Palmadesso, 1986). Such a normal component does not exist in the present case, since the discontinuity is tangential and it remains tangential throughout the development of the $\mathrm{KH}$ instability. However, bending of the magnetic field lines produces similar chaotic consequences for the particle dynamics and allows for a transition between different regimes.

The top panel of Fig. 12 presents the three different possible orbits in a tangential discontinuity before the developement of a KH instability. We consider a magnetic field topology defined by (7) with $y_{0}=$ const in the expression of $\chi$. Orbit 1 corresponds to the adiabatic case, far from the discontinuity, for which the gyromotion is regular and the magnetic moment is conserved. Orbit 2 is also regular, with the integral action being conserved. Orbit 3 is somewhat similar to orbit 2, except that it is characterized by a nearly full 


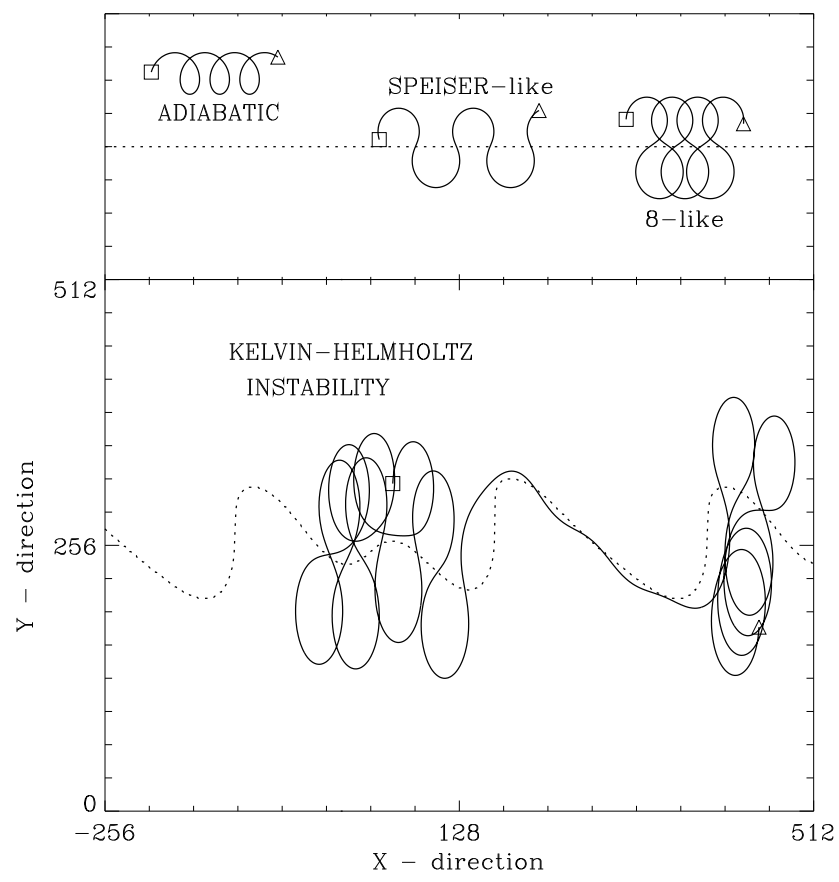

Fig. 12. Different orbit types in a tangential discontinuity: (1) adiabatic, (2) Speiser-like and (3) figure eight-like (upper panel). KH instability essentially allows transition between these $\mathrm{d}$ ifferent types of motion (lower panel).

cyclotron turn in each side of the MP. In the absence of KH instability, particles may exhibit all three behaviors, but retain this character throughout the motion. In contrast, the bottom panel of Fig. 12 shows that the development of a $\mathrm{KH}$ instability and resulting bending of the magnetic field lines results in transitions between these different motions. The mechanism we envision for ions to cross the MP is that the $\mathrm{KH}$ instability enforces transition between different possible regimes in the sharp field reversal region.

As mentioned in Sect. 3, test-particle calculations have been performed in a dimensionless way. The leakage or mass entry process associated with each type of ion needs to be discussed in physical units using realistic values for the MP thickness and the magnetic field magnitude and density in the MSH. With an MP thickness of $0.1 R_{E}, B_{0}=10 \mathrm{nT}$ and $n_{0}=10^{7} \mathrm{~m}^{-3}$ in the MSH, the Alfvén speed is $v_{A}=$ $7 \times 10^{4} \mathrm{~m} \cdot \mathrm{s}^{-1}$. Distances are thus normalized to $1 R_{E}$, velocity to $v_{A}$ and time to $\sim 100 \mathrm{~s}$. With the same values for test-particles calculations, distances are also normalized to $1 R_{E}$, time to $\omega_{c}^{-1}=1 \mathrm{~s}$ and velocity to $6 \times 10^{6} \mathrm{~m} \cdot \mathrm{s}^{-1}$.

Most of the calculations were done with $E=0.05$. For $\mathrm{H}^{+}$ions this corresponds to an energy of $18 \mathrm{keV}$. The associated velocity is $1800 \mathrm{~km} \cdot \mathrm{s}^{-1}$ and the 50 gyroperiods used as the maximum time allowed for the particles to cross the MP correspond to a time-of-flight of $50 \mathrm{~s}$. The shear velocity $\Delta v=2 v_{A}=140 \mathrm{~m} \cdot \mathrm{s}^{-1}$ is one order of magnitude smaller than the particles' velocity and performing the test-particle calculations in the wave frame is thus relevant. Furthermore, since the time-of-flight is smaller than $50 \mathrm{~s}$, the stationary hypothesis is valid as the MHD electromagnetic field weakly evolves on a time scale of $100 \mathrm{~s}$. The assumptions used for the test-particle calculations are justified for $\mathrm{H}^{+}$ions of high energy. Even if Fig. 5 shows that the crossing rate for particles with an initial energy of the order of 0.01 is also of a few percentage points, the results discussed above mainly concern the high energy tail of the $\mathrm{H}^{+}$ion distributions.

When the mass of the particles is increasing, the time normalization increases proportionally to the mass ratio, and the velocity and energy normalization decrease proportionally to the inverse of the mass ratio. This means that the time-offlight increases with particle mass and the velocity decreases. The dimensionless test-particle calculations can also be applied to $\mathrm{He}^{+}$ions. Performing these calculation in the wave frame under the stationary hypothesis is still valid and instead of concerning the high energy tail, it concerns a population closer to the thermal one.

The case of $\mathrm{O}^{+}$ions is different. Considering their large mass, $E=0.05$ corresponds to the thermal population (with an average temparature of $2 \mathrm{keV}$ (see, e.g. Gloeckler and Hamilton, 1987). But then their velocity is about the same as the surface wave and the time-of-flight is about the same as the time scale evolution of the MHD fields. A deeper investigation of the $\mathrm{O}^{+}$ions' case would thus need to take care of these problems, since the temporal evolution of the instability could be important.

Several studies have reported evidences of KH instability at the Earth's magnetopause since the early observation by Lepping and Burlaga (1979). These authors developed a method to infer the amplitude and the wavelength of the $\mathrm{KH}$ instability. Using VOYAGER 1 data, they found an average amplitude of $2100 \mathrm{~km}$ and a wavelength of $47000 \mathrm{~km}$. These observations thus yield a larger value for the $\mathrm{KH}$ instability wavelength, as compared to the one obtained in our simulations. This result is fairly general in numerical simulations of the KH instability. The inverse cascade we observed in the simulations could be one of the reasons for such a disagreement. However, this is of minor importance, since the number of escaping particles per wavelength is somewhat independent of the KH instability wavelength.

In order to explore the dependence upon velocity shear, we performed calculations with $\Delta v=3 v_{A}$. After development of the KH instability, we obtained four wavelengths in the simulation box, that merged into two wavelengths at the end of the simulation. The average value of the CR is $14 \%$, and its standard deviation is $9 \%$. Keeping in mind that with $\Delta v=2 v_{A}$, one has a CR of $12 \%$, with a standard deviation of $5 \%$, we can conclude that the wavelength of the $\mathrm{KH}$ instability is not a predominant parameter. Therefore, it appears that with a velocity shear large enough to ensure the development of a $\mathrm{KH}$ instability, the escaping particle number is anti-correlated with the instability wavelength and the shear velocity.

The results presented above were performed with a high magnetic shear of $180^{\circ}$ that corresponds to a southward IMF. Since MHD simulations require a lot of computer resources, we cannot perform a systematic analysis of the magnetic 
shear influence. Only the $90^{\circ}$ and $135^{\circ}$ magnetic shear cases were considered. It should be noted here that the $X$ component of the magnetic field is important because it has a stabilizing effect for the development of a $\mathrm{KH}$ instability. When the magnetic field is in the same direction as the shear velocity, magnetic tension tends to oppose its bending and thus, has a stabilizing effect. On the other hand, when the magnetic field is normal to the shear velocity, the $\mathrm{KH}$ instability can develop like in a neutral fluid.

In the $90^{\circ}$ magnetic shear case, $\mathrm{KH}$ instability does not develop until the shear velocity reaches $\Delta v=3 v_{A}$. Accordingly, the KH instability cannot drive any substantial MP crossing. From a general point of view, higher values of the velocity shear quickly turn unstable. For a magnetic shear of $135^{\circ}$, the KH instability appears from $\Delta v=3 v_{A}$. The corresponding crossing rate is of a few tenths of percent. This very small value of the crossing rate is due to the nonzero $X$ component of the magnetic field. This component drives the particles along the interface and is responsible for transition toward meandering motion. When the $X$ component of the magnetic field becomes negligible as compared to the $Z$ component, particles that cross the MP have a gyromotion essentially in the $X-Y$ plan and flow along the $Z$ direction. This limits accordingly the number of transient orbits and increases the escape rate.

\section{Conclusion}

Observations of high-speed plasma flows at the MP are generally interpreted as a signature of reconnection. As the outflowing plasma carries substantial magnetic flux, this implies the existence of a magnetic field component (even small) normal to the interface. But under southward IMF, high-speed plasma flows can be observed for which the MP has the structure of a rotational discontinuity (e.g. Sonnerup, 1967; Pashmann et al., 1979; Sonnerup et al., 1981). However, under normal conditions, MSP is closed and MP has the structure of a tangential discontinuity. Considering such a tangential discontinuity, we have shown that the development of a $\mathrm{KH}$ instability at the flanks of the MSP is unable to sufficiently bend magnetic field lines to induce nonadiabatic motion of electrons.

Due to their larger mass, ions are subject to nonresistive diffusion through the MP. The development of a KH instability breaks the regularity of their motion and allows for a net outward flow of these ions. KH instability thus appears as a possible mechanism for the leakage of ions in the MSH or entry of solar wind ions in the MSP. Since the proportion of the concerned population is of a few percentage points, the mass exchange process is not associated with a significant transfer of magnetic flux through the MP.

We have shown that MP crossing does not depend on the velocity shear and leads to a structuring of the escaping population:

1. The escaping rate is barely invariant with the initial particle energy. But the number of particles embeded in the nonadiabatic sheet increases with their energy, and this leads to an outgoing distribution with a high energy tail.

2. The escaping rate depends slightly on the initial pitchangle. However, after crossing of the MP, the outgoing population is peaked in the perpendicular direction.

3. The crossing process is not uniform, and some preferential regions localized at the edge of the wave front of the $\mathrm{KH}$ instability exist.

For a normalized energy of 0.05 , we obtain a crossing rate of $12 \%$; this rate is somewhat independent of the initial energy. The exact value of the crossing rate is meaningless due to the influence of the numerical constraints, but we would like to emphasize that about 1 ion in 10, located in the nonadiabatic sheet (defined above), cross the MP. At this stage, we would like to be able to give an evaluation of the total net mass transfer across the whole MP that results from nonadiabatic motion in a KH instability. We cannot offer this evaluation for essentially two reasons:

- Since the MSH plasma flow is essentially tangential to the MP, it cannot be responsible for bringing particles close to it. Longer time processes (like gradient or curvature drift) can supply particles close to the MP, but the implications of such processes would need relevant calculations or simulations that are far beyond the scope of this paper. On the other hand, large-scale simulations (e.g. Winglee, 1998) that could answer such a problem could not deal correctly with the local effects on particles, such as their nonadiabatic motion.

- Depending on the part of the MP studied, the magnetospheric magnetic field changes in direction and magnitude. Since it is a crucial parameter for the development of a $\mathrm{KH}$ instability, we can definitively not expect that the KH instability would occur on the whole surface of the MP. Once again, unless there is the use of global treatment of the whole MP, it seems unrealistic to evaluate a global mass transfer integrated over the whole cross section surface of the magnetosphere.

Other simulations performed with $90^{\circ}$ and $135^{\circ}$ magnetic shear reveal that the escape rate strongly depends upon the magnetic shear. More specifically, there exists a minimum threshold for the magnetic shear (its value depending on velocity shear) above which a KH instability can develop. Nonresistive diffusion at the MP then occurs due to the nonadiabatic motion of the particles.

Acknowledgements. Part of this work was performed while the first author held a National Research Council-NASA/GSFC Research Associateship. The MHD simulations were done on the Cray C-90 at IDRIS Laboratory, the supercomputing center of CNRS/FRANCE

The Editor in Chief thanks D. Winske and another referee for their help in evaluating this paper. 


\section{References}

Belmont, G. and Chanteur, G.: Advances in magnetopause KelvinHelmholtz instability studies, Phys. Scripta, 40, 124, 1989.

Berchem, J. and Russell, C. T.: The thickness of the magnetopause current layer: ISEE 1 and 2 observations, J. Geophys. Res., 87, 2108, 1982a.

Berchem, J. and Russell, C. T.: Magnetic field rotation through the magnetopause: ISEE 1 and 2 observations, J. Geophys. Res., 87, 8139, 1982b.

Büchner, J. and Zelenyii, L. M.: Regular and chaotic charged particle motion in magnetotaillike field reversals 1 . Basic theory of trapped motion, J. Geophys. Res., 94, 11 821, 1989.

Cahill, L. J. and Amazeen, P. G.: The boundary of the geomagnetic field, J. Geophys. Res., 68, 1835, 1963.

Chandrasekhar, S.: Hydrodynamic and hydromagnetic stability, Dover Publication, 1961.

Chappell, C. R., Moore, T. E., and Waite, Jr., J. H., : The ionosphere as a fully adequate source of plasma for the Earth's magnetosphere, J. Geophys. Res., 92, 5896, 1987.

Chen, J. and Palmadesso, P. J.: Chaos and nonlinear dynamics of single particle orbits in a magnetotaillike magnetic field, J. Geophys. Res., 91, 1499, 1986.

Dungey, J. W.: Interplanetary magnetic field and the auroral zone, Phys. Rev. Lett., 6, 47, 1961.

Eastmann, T. E., Hones, Jr., W., Bame, S. J., and Asbridge, J. R.: The magnetospheric boundary layer: site of plasma, momentum, and energy transfer from the magnetosheath to the magnetosphere, Geophys. Res. Lett., 3, 685, 1976.

Eastmann, T. E. and Hones, Jr., W.: Characteristics of the magnetospheric boundary layer and magnetopause layer as observed by IMP 6, J. Geophys. Res., 84, 2019, 1979.

Fejer, J. A.: Hydromagnetic stability at a fluid velocity discontinuity between compressible fluids, Phys. Fluids, 7, 499, 1964.

Fujimoto, M. and Terasawa, T.: Anomalous ion mixing within and MHD scale Kelvin-Helmholtz vortex, J. Geophys. Res., 99, 8601, 1994.

Fuselier, S. A., Anderson, B. J., and Onsager, T. G.: Particles signatures of magnetic topology at the magnetopause: AMPTE/CCE observations, J. Geophys. Res., 100, 11,805, 1995.

Gloeckler, G. and Hamilton, D. C.: AMPTE ion composition results, Phys. Scripta, T18, 73, 1987.

Gosling, J. T., Asbridge, J. R., Bame, S. J., Feldman, W. C., Paschmann, C., Sckopcke, N., and Russell, C. T.: Evidence for quasi-stationary reconnection at the dayside magnetopause, J. Geophys. Res., 87, 2147, 1982.

Gosling, J. T., Thomsen, M. F., Bame, S. J., Elphic, R. C., and Russell, C. T.: Observation of reconnections of interplanetary and lobe magnetic field lines at the high latitude magnetopause, J. Geophys. Res., 96, 14 097, 1991.

Le, G. and Russell, C. T.: The thickness and structure of high beta magnetopause current layer, Geophys. Res. Lett., 21, 2451, 1994.

Lepping, R. P. and Burlaga, L. F.: Geomagnetopause surface fluctuations observed by Voyager 1, J. Geophys. Res., 84, 7099, 1979.

Midgley, J. E. and Davis, L.: Calculation by a moment technique of the perturbation of the geomagnetic field by the solar wind, $\mathrm{J}$. Geophys. Res., 68, 5111, 1963.

Mitchell, D. G., Kutchko, F., Williams, D. J., Eastman, T. E., Franks, L. A., and Russell, C. T.: An extended study of the lowlatitude boundary layer on the dawn and dusk flanks of the magnetosphere, J. Geophys. Res., 92, 7394, 1987.

Miura, A. and Pritchett, P. L.: Nonlocal stability analysis of the
MHD Kelvin-Helmholtz instability in a compressible plasma, J. Geophys. Res., 87, 7431, 1982.

Miura, A.: Simulation of the Kelvin-Helmholtz instability at the magnetospheric boundary, J. Geophys. Res., 92, 3195, 1987.

Miura, A.: Self-organization in the two-dimensional magnetohydrodynamic transverse Kelvin-Helmholtz instability, J. Geophys. Res., 104, 395, 1999.

Moore, T. E. and Delcourt, D. C.: 'The Geopause', Rev. Geophys., 33, 175, 1995.

Papamastorakis, I., Paschmann, G., Sckopke, N., Bame, S. J., and Berchem, J.: The magnetopause as a tangential discontinuity for large field rotation angles, J. Geophys. Res., 89, 127, 1984.

Paschmann, G., Sonnerup, B. U. Ö., Papamastorakis, J., Bame, S. J., Asbridge, J. R., Gosling, J. T., Hones, Jr., E. W., and Tech, E. R.: ISEE plasma observations near the subsolar magnetopause, Space Sci. Rev., 22, 717, 1978.

Paschmann, G., Sckopke, N., Haerendel, G., Papamastorakis, I., Sckopke, N., Haerendel, G., Bame, S. J., Asbridge, J. R., Gosling, J. T., Russell, C. T., and Elphich, R. C.: Plasma acceleration at the Earth's magnetopause: evidence for magnetic reconnection, Nature, 282, 243, 1979.

Paschmann, G., Papamastorakis, J., Baumjohann, W., Sckopke, N. K., Carlson, C. W., Sonnerup, B. U. Ö., and Lühr, H.: The magnetopause for large magnetic shear: AMPTE/IRM observations, J. Geophys. Res., 91, 11 099, 1986.

Paschmann, G., Baumjohann, W., Sckopke, N. K., Phan, T. D., and Lühr, H.: Structure of the dayside magnetopause for low magnetic shear, J. Geophys. Res., 98, 13 409, 1993.

Phan, T. D., Paschmann, G., Baumjohann, W., Sckopke, N., and Lühr, H.: The magnetosheath region adjacent to the dayside magnetopause: AMPTE/IRM observations, J. Geophys. Res., 99, 121, 1994.

Phan, T. D. and Paschmann, G.: Low-latitude dayside magnetopause and boundary layer for high magnetic shear 1. structure and motion, J. Geophys. Res., 101, 7801, 1996.

Phan, T. D., Paschmann, G., and Sonnerup, B. U. Ö.: Low-latitude dayside magnetopause and boundary layer for high magnetic shear 2. occurence of magnetic reconnection, J. Geophys. Res., 101, 7817, 1996.

$\mathrm{Pu}, \mathrm{Z}$. Y. and Kivelson, M. G.: Kelvin-Helmholtz instability at the magnetopause: solution for a compressible plasma, J. Geophys. Res., 88, 841, 1983.

Scurry, L., Russell, C. T., and Gosling, J. T.: A statistical study of accelerated flow events at the day side of the magnetopause, J. Geophys. Res., 99, 14 815, 1994.

Sen, A. K.: Effects of compressibility on a Kelvin-Helmholtz instability in a plasma, Phys. Fluids, 7, 1293, 1964.

Shelley, E. G., Peterson, W. K., Ghielmetti, A. G., and Geiss, J.: The polar ionosphere as a source of energetic magnetospheric plasma, Geophys. Res. Lett., 9, 941, 1982.

Smets, R.: Charged particle dynamics in a tangential discontinuity, J. Geophys. Res., 105, 25 009, 2000.

Song, P., Russell, C. T., Fitzenreiter, R. J., Gosling, J. T., Thomsen, M. F., Mitchell, D. G., Fuselier, S. A., Parks, G. K., Anderson, R. R., and Hubert, D.: Structures and properties of the subsolar magnetopause for northward IMF: multiple-instrument observations, J. Geophys. Res., 98, 11 319, 1993.

Sonnerup, B. U. Ö.: Adiabatic particle orbits in a magnetic null sheet, J. Geophys. Res., 76, 8211, 1971.

Sonnerup, B. U. Ö., and Cahill, L. J.: Magnetopause structure and attitude from Explorer 12 observations, J. Geophys. Res., 72, $171,1967$. 
Sonnerup, B. U. Ö., Paschmann, G., Papamastorakis, I., Sckopke, N., Haerendel, G., Bame, S. J., Asbridge, J. R., Gosling, J. T., and Russell, C. T.: Evidence for magnetic reconnection at the Earth's maghnetopause, J. Geophys. Res., 86, 10 049, 1981.

Southwood, D. J.: The hydromagnetic stability of the magnetospheric boundary, Planet. Space Sci., 16, 587, 1968.

Speiser, T. W.: Particle trajectory in model current sheets, 1, Analytical solutions, J. Geophys. Res., 70, 4219, 1965.

Talwar, S. P.: Hydromagnetic stability of the magnetospheric boundary, J. Geophys. Res., 69, 2707, 1964.

Terasawa, T., Fujimoto, M., Karimabadi, H., and Omidi, N.: Anomalous ion mixing within a Kelvin-Helmholtz vortex in a collisionless plasma, Phys. Rev. Lett., 68, 2778, 1992.

Thomas, V. A.: Three-dimensional kinetic simulation of the KelvinHelmholtz instability, J. Geophys. R., 100, 19429, 1995.

Thomas, V. A. and Winske, D.: Kinetic simulation of the KelvinHelmholtz instability at the magnetopause, J. Geophys. Res., 98, $11425,1993$.

Walker, A. D. M.: The Kelvin-Helmholtz instability in the low latitude boundary layer, Planet. Space Sci., 29, 1119, 1981.

Winglee, R. M.: Multifluid simulations of the magnetosphere: the identification of the geopause and its variation with IMF, Geophys. Res. Lett., 25, 4441, 1998. 